\title{
Smart COVID-shield: an loT driven reliable and automated prototype model for COVID-19 symptoms tracking
}

\author{
Hrudaya Kumar Tripathy ${ }^{1}$ (D) Sushruta Mishra ${ }^{1}$ (D) Shubham Suman ${ }^{1}$. \\ Anand Nayyar ${ }^{2}$ (D) $\cdot$ Kshira Sagar Sahoo ${ }^{3}$
}

Received: 19 May 2021 / Accepted: 28 November 2021 / Published online: 18 January 2022

(c) The Author(s), under exclusive licence to Springer-Verlag GmbH Austria, part of Springer Nature 2021

\begin{abstract}
IoT technology is revolutionizing healthcare and is transforming it into more personalized healthcare. In the context of COVID-19 pandemic, IoT's intervention can help to detect its spread. This research proposes an effective "Smart COVID-Shield" that is capable of automatically detecting prevalent symptoms like fever and coughing along with ensuring social distancing norms are properly followed. It comprises three modules which include Cough Detect Module (CDM) for dry cough detection, Temperature Detect module (TDM) for high-temperature monitoring, and Distance Compute Module (DCM) to track social distancing norm violator. The device comprises a combination of a lightweight fabric suspender worn around shoulders and a flexible belt wrapped around the waist. The suspender is equipped with a passive infrared (PIR) sensor and temperature sensor to monitor persistent coughing patterns and high body temperature and the ultra-sonic sensor verify 6 feet distance for tracking an individual's social distancing norms. The developed model is implemented in an aluminum factory to verify its effectiveness. Results obtained were promising and reliable when compared to conventional manual procedures. The model accurately reported when body temperature rises. It outperformed thermal gun as it accurately recorded a mean of only 4.65 candidates with higher body temperature as compared
\end{abstract}

\footnotetext{
Anand Nayyar

anandnayyar@duytan.edu.vn

Hrudaya Kumar Tripathy

hktripathyfcs@kiit.ac.in

Sushruta Mishra

sushruta.mishrafcs@kiit.ac.in

Kshira Sagar Sahoo

kshirasagar12@gmail.com

1 School of Computer Engineering, KIIT Deemed To Be University, Bhubaneswar, Odisha, India

2 Graduate School, Faculty of Information Technology, Duy Tan University, Da Nang 550000, Vietnam

3 Department of CSE, SRM University, Amaravati, AP, India
} 
to $8.59 \%$ with the thermal gun. A significant reduction of $3.61 \%$ on social distance violators was observed. Besides this, the latency delay of $10.32 \mathrm{~s}$ was manageable with the participant count of over 800 which makes it scalable.

Keywords Internet of Things (IoT) · COVID-19 · Smart COVID-shield · Cough detect module (CDM) · Temperature detect module (TDM)

Mathematics Subject Classification 68-04 · 68T09 • 68T42

\section{Introduction}

At present global society is experiencing a major threat due to COVID-19 crisis [1]. From early 2020 this has created a havoc and panic situation. This has not only challenged the health dynamics of a nation but also, the economic conditions of the entire world [2, 3]. The first confirmed scenario came from the capital of Wuhan, China and since then the virus had resulted in massive loss of life before declaring it as a global threat $[4,5]$. This has diffused to around 141 nations causing massive loss of life around the world [6]. Some prevalent symptoms include high body temperature, persistent coughing, and fatigue or other cases, affected people may feel pains \& sore throat, congestion in nasal passages, running nose with diarrhea [7-9]. However, there are observed cases when the infected person may not witness any immediate common symptoms. It is quite alarming particularly for elderly mass or a patient suffering from serious disease risks and it develops breathing difficulty for them. Potential vaccines and some clinical-based treatments are still under scrutiny which is under observation and are tested by renowned healthcare and drug research firms. Subsequently, efforts are in progress by WHO to generate curative drugs for effective treatment of COVID19. With the rise in death cases due to pandemics, several countries have imposed lockdown to restrict the rapid spread of the risks. Rigorous social distancing norms are being strictly imposed in many regions. Still, in a densely populated area, it is almost impossible to oversee that these guidelines are strictly followed. They also, try to identify the infection among the crowd by screening the temperature in public places using infrared thermometer. But the effectiveness of the infrared thermometer gun is questionable as it fails to cover a larger geographical region and also, consumes more time. Besides this, continuous dry coughing is another observed dominant symptom that is cumbersome to monitor in a crowded place. Hence a more efficient symptom monitoring alternative can resolve the flaw.

The implementation of the IoT in the smart city has showcased its strength right from the introduction of the concept of the smart city [10]. IoT revolution is remodeling modern healthcare with the incorporation of new technologies from both social and economic prospects. It is also, bringing a constructive paradigm shift from the traditional healthcare system to a more personalized space. It is setting up a benchmark in delivering healthcare models and services at cost-effective rate thereby offering a better quality of services with its advanced user experience [11]. Due to its wide range 


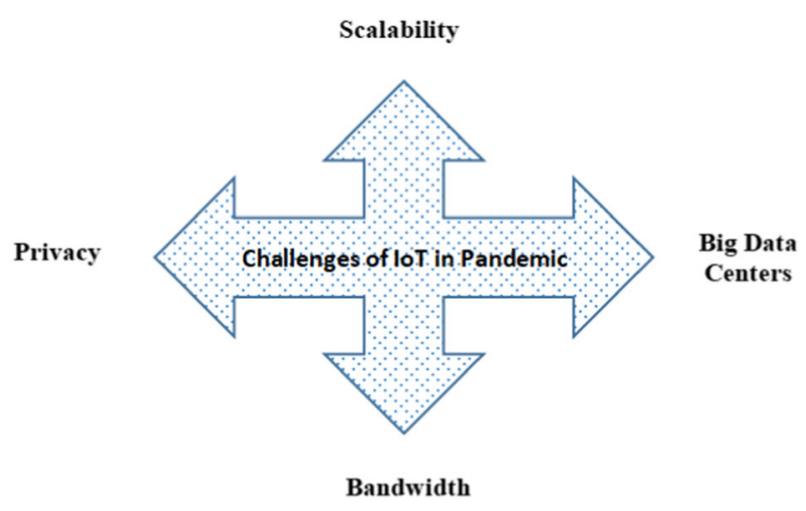

Fig. 1 Challenges of IoT in pandemic scenario

of capabilities like object tracking, identification, authentication and data aggregation among some of them, it makes the best suitable technology stack in the field of healthcare [12].

During this COVID-19 pandemic period, researchers are taking benefit of different technologies and IoT is one of the most preferred among them. Though it offers several benefits still there are some basic challenges faced by IoT concerning this pandemic as depicted in Fig. 1. In the context of monitoring the pandemic situation, IoT can be considered to be safe and efficient in tracking potential suspects with COVID-19 symptoms risks. Symptoms which include a persistent dry coughing and high body temperature are verified by healthcare experts. Continuous coughing for $15 \mathrm{~s}$ or exhibiting a higher body temperature beyond 37 degrees Celsius is perceived as an alarming risk of COVID-19 risks. Besides this, everywhere government strictly recommends following a social distancing norm where at least a 6 feet distance is supposed to be maintained among individuals at public places. This minimizes the probability of infection but it has been observed that in many regions, people are violating the social distancing norms as well as not taking the symptoms seriously. This is a very serious issue that needs to be taken care of. Especially in crowded places, it is a big challenge to monitor many people randomly gathered in a big way. This poses a serious challenge for concerned officials to keep track of the public in these regions on a regular basis. In such a scenario, a smart IoT-based deployment can be of big help in regulating social distancing norms along with people with common symptoms like high body temperature and persistent cough can be detected. Thus, efficient IoT-based technology can be useful in assisting patients, healthcare support staff, and concerned authorities to track corona virus affected people among the huge mass.

The diagrammatic view of the risk factors associated with COVID-19 detection is shown in Fig. 2. Based on these factors, an automated smart deployment model for COVID-19 symptom detection is developed which is named as "Smart COVID Shield". Any organization that is willing to use this application must register its employees in this application to generate a unique identifier. This smart model uses three distinct functioning units one each for coughing detection, high body temperature 
COMMON SYMPTOMS (\%) OF COVID-19

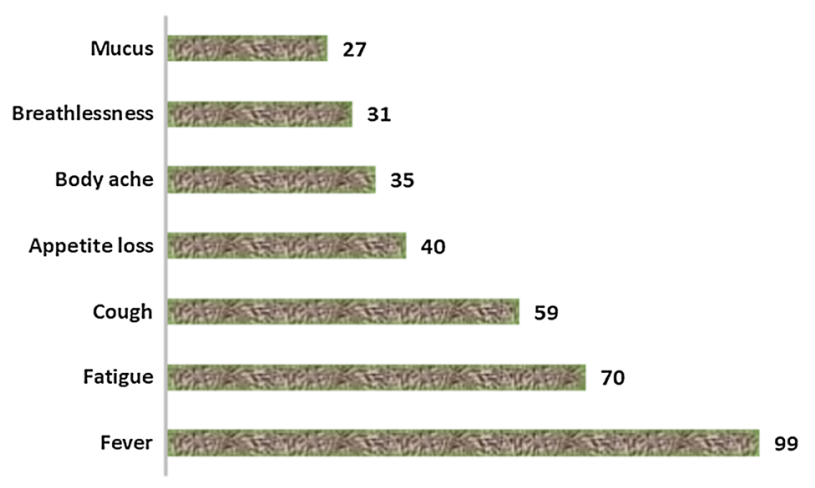

Fig. 2 Common relevant symptom analysis of COVID-19

identification, and social distancing norm violation. If any ambiguity and violation on basis of these three functioning units are detected in any person by admin then the IDs of those users are collected and along with its parameters and details of that users are recorded which is eventually notified to the admin in real-time. Admin is responsible for forwarding the gathered information to nearby medical centers for assistance and support.

The main contributions of the research work are listed as follows:

a. Persistent dry coughing and abnormally high body temperature are identified as more relevant risk factors associated with COVID-19 crisis and are analyzed in the study. Besides these, maintaining 6 feet social distancing norm was also recognized as a crucial COVID-19 parameter.

b. Taking these three features into consideration, designing a smart, reliable and efficient COVID-19 tracking device model to monitor suspected infected people at public places forms the core research work.

c. The device named as "Smart COVID-Shield" is equipped with a suspender and a belt to be placed over clothes of the user which constitutes Cough Detect Module (CDM), Temperature Detect module (TDM) and Distance Compute Module (DCM).

d. Coughing and temperature patterns can be detected through PIR sensor of the suspender while a belt with ultrasonic sensor can be used to track people who violate the 6 feet social distancing norms in real-time environment. Further, the detected patterns can be continuously monitored and communicated through GPS and GSM unit to health units in case of discrepancy.

e. "Smart COVID-Shield" is deployed in an aluminum factory and was utilized by users of varying age groups. Social distancing violation was accurately verified. The model's performance experimentally outperformed that of the thermal gun for high-temperature detection. High coughing activity of users was also accurately validated. 
f. A significant drop in social distancing norm violators was recorded over its period of implementation with increased awareness among public. Apart from these, in crowded places it could produce results and give accurate analysis in quick time with less latency making it more scalable as well as reliable.

The rest paper is arranged as follows. Section 2 highlights the important existing works and models developed by different authors during the ongoing crisis. Section 3 describes the proposed working model and its different functionalities in detail. Section 4 discusses the implementation results after deploying the working model in real-time scenario. Finally, Sect. 5 concludes the work with a glimpse of the future scope of research.

\section{Literature survey}

Since the beginning of the year 2020, the world is experiencing a struggling phase with the pandemic caused by severe infectious disease. To control the spread of the virus various works are going on the vaccine but many works are rapidly taking place in developing such gears to help in curbing the virus. As per the tally shows no treatment has showcased promising results in stopping the virus so, a monitoring device is needed [13]. Many patients with mild symptoms have recovered from the disease while staying at home, but that does not guarantee the safety of getting it reinfected. Taking the case of reinfection, the chances of returning the symptoms is very high. Thus, to prevent such infectious diseases from spreading social distancing and proper sanitization should be maintained [14]. IoT technology during the COVID-19 has displayed its usefulness in assisting patients, healthcare providers, and authorities.

In this section, researchers have tried to briefly highlight various relevant services facilitated by IoT and its usage in various ways possible including drones, wearables, robots, and applications for smartphones [15]. These technologies are mainly utilized by the frontliners for combating COVID-19. A comparative analysis of the benefits and limitations of different models employed during this pandemic is highlighted in Table 1.

Wearable technology is the latest tech gaining much popularity because of its immense performance ability as well as these are being used as styling gears. Wearable techs are any electronic sensor or devices which are combined in such a way that it can be worn by people. It is an application configured device that takes up data from the device as input from the wearable gadget in the form of bands, sticks, glasses, belts, etc., and then processes the data for better output delivery. There is a wide range of wearable devices such as Smart Helmets, Smart Thermometers, Smart Glasses, EasyBand, and Proximity Trace. Table 2 highlights some widely popular wearables used worldwide to deal with a pandemic.

The modernized phone services are the applications framed for limited functionalities. Many effective mobile usage are designed and applied to clinical sector while few are displaying its concerns towards the pandemic as presented in Table 3.

Drones are simply flying objects which are without any human beings inside it, it is operated by a human being by remote monitoring. The implementation of drones 
Table 1 Pros and cons of devices used during pandemic

\begin{tabular}{|c|c|c|}
\hline Model & Benefits & Limitations \\
\hline Wearables & $\begin{array}{l}\text { Regular Monitoring } \\
\text { Improved Patient's Medicare } \\
\text { Reduced clinical visit }\end{array}$ & $\begin{array}{l}\text { Data security and privacy } \\
\text { Less battery life span }\end{array}$ \\
\hline Smart phones & $\begin{array}{l}\text { Ease of tracking and monitoring } \\
\text { Cost minimization }\end{array}$ & Gathered data security an issue \\
\hline Drones & $\begin{array}{l}\text { Searching and delivering with ease } \\
\text { Reachability to remote sites } \\
\text { Less worker physical interactions }\end{array}$ & $\begin{array}{l}\text { Quality of service uncertain } \\
\text { Lower connectivity } \\
\text { Less security in unstructured data }\end{array}$ \\
\hline Robots & $\begin{array}{l}\text { Remote treatment and diagnosis } \\
\text { Cleaning and disinfecting at ease }\end{array}$ & $\begin{array}{l}\text { Privacy concerns } \\
\text { Mental health issues }\end{array}$ \\
\hline
\end{tabular}

Table 2 Some popular wearables used during pandemic

\begin{tabular}{|c|c|c|}
\hline Model & Purpose & Examples \\
\hline Smart Thermometers[16] & Temperature monitoring & $\begin{array}{l}\text { Kinsa, Tempdrop, } \\
\text { iSense }\end{array}$ \\
\hline Smart Helmet [17] & $\begin{array}{l}\text { Temperature regulation, coordinate and face } \\
\text { detection }\end{array}$ & KC N901 in China \\
\hline Smart Glasses [18] & Track high body temperature and regulation & $\begin{array}{l}\text { Rokid in China } \\
\text { Vuzix \& Onsight }\end{array}$ \\
\hline IoT-Q-Band [19] & $\begin{array}{l}\text { Tracking quarantined objects in case of } \\
\text { absconding }\end{array}$ & $\begin{array}{l}\text { Hong Kong electronic } \\
\text { wristband }\end{array}$ \\
\hline EasyBand [20] & Social distance norms tracking & Pact wristband \\
\hline Proximity Trace [21] & Tracing contacts of contaminated employee & Hardhat TraceTag \\
\hline
\end{tabular}

Table 3 Some popular smart phone applications used during pandemic

\begin{tabular}{ll}
\hline Model & Purpose \\
\hline $\begin{array}{l}\text { Aarogya Setu [22] } \\
\text { NCapp [23] }\end{array}$ & $\begin{array}{l}\text { Interconnecting public with medical services } \\
\text { Provide available consulting }\end{array}$ \\
Detecta Chem [24] & $\begin{array}{l}\text { Enabling COVID-19 tests accompanied by a cost effective kit designed with } \\
\text { mobile phone functionalities }\end{array}$ \\
Stop Corona [25] & $\begin{array}{l}\text { Developing a coordinate axis along with optimal risk sites } \\
\text { Selfie app [26] }\end{array}$ \\
Civitas [27] & $\begin{array}{l}\text { Detecting best time for potential suspects } \\
\text { TraceTogether [28] }\end{array}$ \\
Coaltion [29] & User's data access through government officials \\
Hamagen [30] & Decurely informing detected cases with users contacts
\end{tabular}


with IoT is known as the Internet of Drone Things (IoD). IoD makes a variety of tasks possible such as tracing, monitoring, delivering, spying, etc. Smart drones can be managed by smartphones with minimum time frame and energy, which makes them more efficient in different fields such as agriculture, military, healthcare, etc. There are also, some drones having thermal imaging facility which makes them suitable for medical and warfare drones. Thus, drones can also show up their potential in fighting against corona. Some widely used drone services are shown in Table 4.

An assembled machine that resembled a living species with an independent movement capability can be called a s a robot. With the rise of inter-networking robots in this cloud computing age, the IoT can be integrated with robot to perform variety of complicated tasks to make things simpler. In context of the present pandemic situation, these robots may be classified into telerobots, social robots, collaborative robots and auto-robots. Popular robotic based applications are depicted in Table 5.

As it is obvious that the world is witnessing a blockage on day-to-day movements. Many social as well as economic backbone institutions, firms, schools, colleges and

Table 4 Some popular drones based services used during pandemic

\begin{tabular}{|c|c|c|}
\hline Model & Purpose & Examples \\
\hline Thermal Imaging Drone [31] & $\begin{array}{l}\text { Temperature capturing in the } \\
\text { crowd }\end{array}$ & Pandemic Drone \\
\hline Disinfectant Drone [32] & Sterilizing contaminated areas & DJI \\
\hline Medical/Delivery Drone [33] & Reducing the hospital visits & Delivery Drone Canada \\
\hline Surveillance Drone [34] & $\begin{array}{l}\text { Crowd social distancing } \\
\text { monitoring }\end{array}$ & Micro Multi-Copter \\
\hline Announcement Drone [35] & $\begin{array}{l}\text { Broadcasting information about } \\
\text { COVID-19 }\end{array}$ & $\begin{array}{l}\text { Broadcasting drone in Spain and } \\
\text { Kuwait }\end{array}$ \\
\hline Multipurpose Drone [36] & $\begin{array}{l}\text { Temperature capturing, } \\
\text { Disinfecting areas, Crowd } \\
\text { monitoring, Broadcasting } \\
\text { information }\end{array}$ & Corona Combat \\
\hline
\end{tabular}

Table 5 Some popular robots used during pandemic

\begin{tabular}{llc}
\hline Model & Purpose & Examples \\
\hline Auto-Robot [37] & $\begin{array}{l}\text { Detecting symptoms, regulating social distancing, } \\
\text { avoiding infections of health personnel, sanitizing } \\
\text { with sterilizing affected sites in health centers, } \\
\text { validating breathing rate of patients } \\
\text { Minimizing infection risk for health staffs }\end{array}$ & $\begin{array}{c}\text { Intelligent Care } \\
\text { Robot }\end{array}$ \\
$\begin{array}{l}\text { Telerobots [38] } \\
\begin{array}{l}\text { Collaborative Robots } \\
\text { Social Robot [40] }\end{array}\end{array}$ & Lower healthcare workers' fatigue & $\begin{array}{c}\text { DaVinci surgical } \\
\text { robot }\end{array}$ \\
& Reducing mental strain & $\begin{array}{c}\text { Asimovotics } \\
\text { Paro }\end{array}$ \\
\hline
\end{tabular}


offices is facing hindrance during this time. As technology has been always seen as the savior of the mankind, researchers have also, dived with devotion to fight this deadly and severely spreading disease. The ability of IoT technology and the strength of smartphones motivated the researchers to move forward and come out with the solution of this problem. Throughout this pandemic, numerous devices and models have been developed. Most of these services serve the purpose of monitoring and tracking of COVID-19 risks. Various works by different authors are highlighted here. Many developed modules and devices lack robustness and reliability. In few cases, privacy of data may be compromised while in several models, portability is an issue. In certain cases, adequate quality of service is not available while prolonged connectivity is also, a concern for some. Thus, keeping in view of these concerns and limitations, a smart and reliable IoT based robust model has been developed in this study for efficient monitoring and tracking of COVID-19 symptoms in public places. The IoT sensor deployed in the model prototype are capable of taking feedback from the body like continuous body motion during cough, body temperature and also, whether the social distance is being maintained or not. The pandemic in itself had a huge impact on human beings adversely so, in order to do a bit to curb this virus spread, the researchers did their bit to demonstrate such a device calibrated with central server of the organization which will deploy the model to fight against the infectious disease.

\section{Proposed smart COVID-shield model}

This section presents the proposed smart prototype designed for COVID-19 tracking and handling. Effective monitoring of people be done if its more relevant and common risk factors can be detected at an early phase. Hence more prevalent symptoms of corona virus need to be tracked and figured out before it gets too late. Symptoms usually begin to show few days after coming into contact with the virus. Common factors like fever, fatigue, cough and others have been identified as general symptoms shown in Fig. 2. It is observed that maximum patients infected with this virus shows higher body temperature, fatigue and cough. An approximate population exhibiting fever and cough roughly estimates to around $99 \%$ and $59 \%$ respectively indicated that high body temperature and persistent coughing activity has been identified as the two most common symptoms in COVID-19 patients [41-43].

Besides these two symptoms, social distancing is another concept that is imposed in almost all nations worldwide. It is declared and proved by WHO that people maintaining a distance of at least 6 feet among themselves in public places has a less probability of getting infected from the virus. In our research these three factors which include high body temperature, persistent cough and social distancing are taken into consideration for COVID-19 tracking.

A new smart, real time and reliable tracking prototype device model is developed that incorporates these three factors into it and helps in effective monitoring of potential people showing early signs of COVID-19. The developed device is named as "Smart COVID-Shield". The graphical prototype view of the proposed work with the above factors under consideration is illustrated in Fig. 3. 


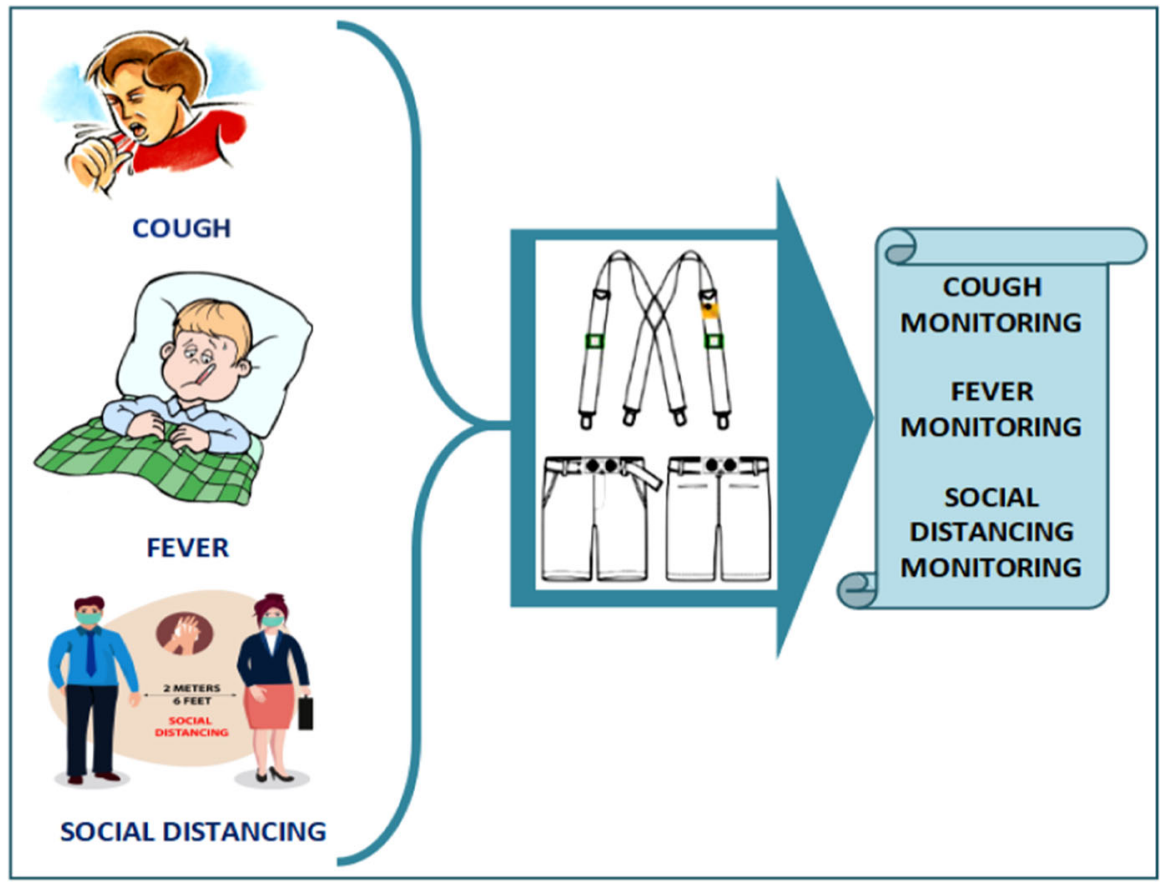

Fig. 3 A prototype of the proposed model handling identified symptoms

\subsection{System requirements}

Various hardware and software requirements are the prerequisite for the working of the model. These are presented in this section.

Passive Infrared Sensor (PIR sensor): This is a motion detecting sensor which determines infrared light that radiates from objects in its range. Here it is used to raise alert notification when persistent coughing activity in a user is recorded.

Temperature Sensor: A temperature sensor transforms the input data into electronic record and is used in this work to continuously measure the body temperature of the user and notifies in case of fever and any abnormal variation in temperature.

Ultrasonic Sensor: An ultrasonic sensor acts as an electronic unit to measure the desired object distance by ultrasonic wave emission which then maps the sound wave onto signal. Here it is utilized to detect people who violate the 6 feet social distance norms.

Arduino Board: It is an open-source cross-platform interface comprising hardware and software embedded in it. It is capable of reading an input, activating a sensor or button or notifying a message and thereby transforming it into desired action.

Connecting Jumper Wires: Jumper wires are used to interconnect various objects on arduino board and header pins without soldering.

Punch-button: A plastic punch-button is included in the model which acts as a push button which is used to switch on the arduino board and activate the overall system. 
Belt: Here a light weight flexible fabric strap is worn around the waist and it is equipped with 4 ultrasonic sensor in four different directions. It is useful in monitoring people in public places who tend to violate 6 feet social distance norms.

Suspender: In this model, a pair of fabric elastic suspender is attached around the shoulders and is worn over clothes. PIR senors are attached to it to detect persistent dry cough patterns and also, temperature sensor is integrated to it to monitor fever and abnormal high body temperature.

Arduino Integrated Development Environment (IDE): This is the open source software interface is used for coding, execution and uploading purpose. Arduino IDE 2.0 beta is used in our work as it is fast and powerful. The platform is more responsive with features like auto-completion and live debugging.

The overall circuit view highlighting the architectural components like sensor, hardware and software is depicted in Fig. 4. The overall circuit model formulated in this work denotes the proper utilization of resources and the equipment's are interlinked for precise functioning of the device. The modules used in the model includes battery, push button, Arduino UNO, ultrasonic sensor, PIR sensor and temperature sensor. The battery powers the entire system which is triggered by the push button attached to it. The power is then transferred to the Arduino board which then analyses the signals received from the ultrasonic sensor, the temperature sensor and the PIR sensor. Module mounted over the belt tracks and validates whether the social distance norm is followed or not and accordingly the feedback is given to the Arduino board. Same operational scenario exists with the temperature sensor and the PIR sensor too. They take the body vitals as input which get validated by the arduino board. After processing of all body vitals, the board finally generates the output as a warning audio signal so as to maintain social distance. The LED bulb glows to indicate an increase in body

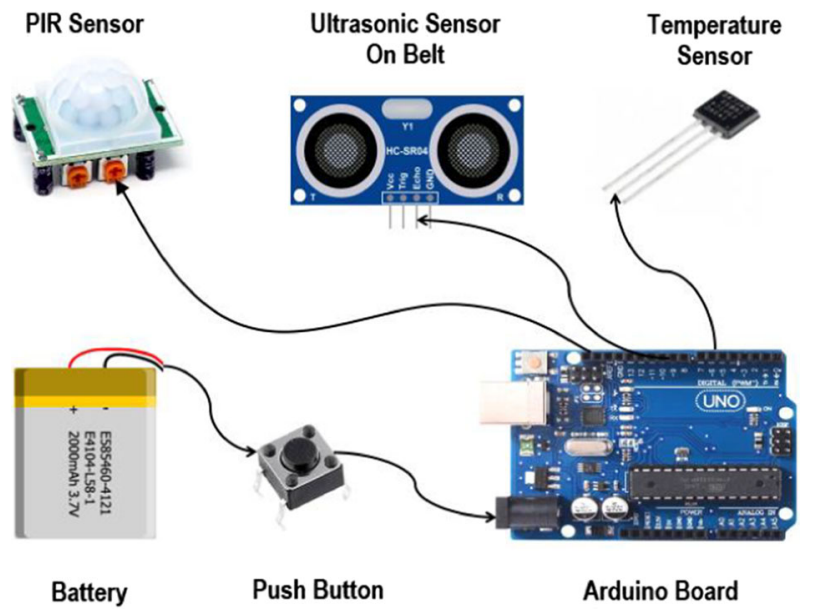

Fig. 4 Circuit diagram of the proposed work 


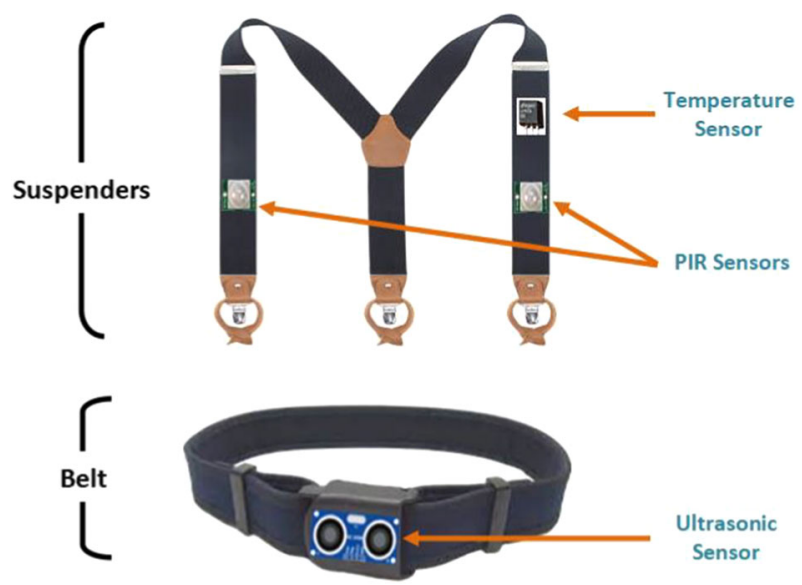

Fig. 5 Skeleton view of proposed suspender and belt equipped with sensor

temperature of the device bearer and sends all the information to a centrally monitored management.

\subsection{Working methodology and functionalities}

The proposed work constitutes the design of a smart IoT enabled COVID-19 symptoms monitoring framework. Primarily persistent cough and high body temperature are the critical symptoms identified in the research. Also, the 6 feet social distancing norm is the third criteria considered in the study. Ultrasonic sensor and PIR sensor are basically used in tracking the symptoms and detecting social distance norm violators. A skeleton model of the suspender and belt is shown in Fig. 5. Four ultrasonic sensor are deployed in belt in all four directions to track social distance violators and it is used within suspender to monitor temperature fluctuations in user. While PIR sensor which are embedded in suspender are utilized to detect persistent dry cough patterns in user.

The 'Smart COVID-Shield' can be easily worn over clothes and is easy to use. The user can use it when stepping outside in public crowded places. It can be logically partitioned into three sub constituents which include CDM to detect cough pattern, TDM to track high body temperature and DCM to trace social distancing norm violators in crowd. TA sample view of the device is shown in Fig. 6.

The detail functionalities of individual modules are discussed here. Cough Detect Module (CDM), Temperature Detect Module (TDM) and Distance Compute Module (DCM) are the three different modules integrated in the model. A persistent cough of more than $15 \mathrm{~s}$ is automatically detected by CDM. A continual higher body temperature beyond 37 degrees Celsius is tracked by TDM. People violating the 6 feet social distancing norm is alerted through DCM.

Cough has been marked as the one of the major symptoms of Covid-19. The CDU is the part of device which takes up the record of cough by any individual. The CDU is 


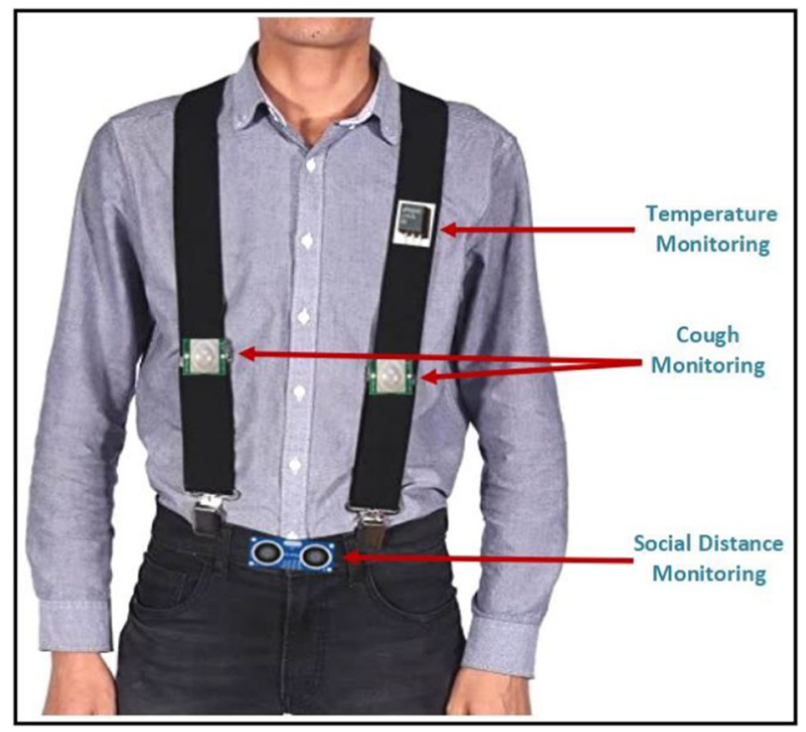

Fig. 6 Smart COVID-Shield embedded with suspender and belt

designed such a way that it gets mounted easily over the dress the same as a suspender is worn. The device consists of PIR sensor. The job of PIR sensor is to take up the body motion of the individual wearing this device. There are two PIR sensor mounted on the chest level to accumulate the data. Here the data is collected as the user coughs, that means that whenever the user coughs his body comes into an upside and downside motion for a while. Hence, if the phenomenon is repeated consistently for a time series or fraction of seconds then it is a matter of concern and precaution should be taken. The cough data is then transferred to the Arduino board for analysis. The Arduino board is responsible for all sorts of analysis and decision making as its the heart of the device. Here a decision is done for a number of coughs and its time frame. The rule for such decision is that if the Persistent Cough Span (PCS) period is more than $15 \mathrm{~s}$ and less than $30 \mathrm{~s}$ then it's a serious issue which should be reported to the authorities. After the decision, the location data and ID of the device is taken by the help of GPS module incorporated with the device. These information's are then forwarded to the GSM module for forwarding the data to the centralized database. The centralized database holds all the records of the user who violates any norms of Covid-19 precaution or has symptoms of it. The data in the database are easily accessible to the authorities of an institution to keep a track over their employees, students and other staffs. So, after this stage the governing bodies can immediately spread this news to the nearby Covid hospitals for check-up of that user.

Temperature is also, one of the major symptoms studied so far for this Covid-19. The temperature detect unit us the unit of the device which takes the body temperature details of the user. According to studies this is the simplest way of tracing any sort of ailment which can further be taken for other precaution measures. The temperature of the user is measured by a temperature module. The module is mounted on the suspender 
in such a manner that it will take up the body temperature same as the thermometer placed by the doctors. The temperature data is sent by the module to the arduino board where the analysis is done. The Arduino board checks whether the body temperature of the user is violating its rule or not. The rule for the temperature detection unit is that the body temp should not be greater than $37^{\circ}$ centigrade. Whenever the user's body temperature exceeds 37 centigrade, a red led glows at the chest level of the user which makes him/her as well as the authority and the neighbour alert. The geo-location data is taken by GPS module and forwarded to the centralized database with the use of GSM module. This helps the authorities of the institution to take serious note of the user and forward the details to the local hospital to take preventive measure.

The social distance is the key preventive measure which can be taken to curb the widespread of the infectious virus. Many government initiatives have been taken for promoting the implementation of social distancing. Social distancing will and is showcasing its effectiveness in breaking the chain of virus. Here the unit is a most important part of the device. The unit is mounted over a belt which is a common accessory for any human being. The placement of Ultrasonic sensor all around the belt for maintaining 6 feet distance in 360 degrees which makes it more acceptable in the market. All four ultrasonic sensor which keeps a track of all distance management. The sensor transmits a signal and if the signal strikes anybody before 6 feet it will make a beep sound to alert the user as well as the others to take a distance apart from the person. Any distance defaulters, their ID is taken by the GPS and GSM module to the centralized database and which can be operated by the higher authorities of any Institution. The data from the centralized database can be forwarded to the nearby hospitals for future preventive measures.

The functional modules and the overall graphical model is presented in Fig. 7. The developed smart model for COVID-19 detection is transformed into a convenient graphical interface view. The work flow denoting the graphical interface of the model is represented in Fig. 8. Primarily the model comprises five distinct functional units.

- General-Info Unit: It acts as the welcome page which introduces users regarding the smart application. The term 'Smart COVID Shield' is written in this welcome page along with the general information such as date and purpose of its development.

- Admin-Login Unit: This unit is exclusively reserved for admin personnel. The admin of the organization that make use of the application has the access for this functional module. A unique user name and password credentials are provided to the admin. Using those security credentials, he can log into the application and operate it as per the requirement. All vital information such as ID of persons who are using the application on any specific date, cough pattern data of registered users, high temperature data and social distance violation information are accessible to the admin.

- Dashboard Unit: All required information for any particular day are stored in data store and displayed in the dashboard which is only accessible to the admin. It records IDs of all persons who violated social distance norms, persons with suspicious cough patterns and persons detected with high temperature. This information is regularly updated and flashed over the smart and automated dashboard in every 


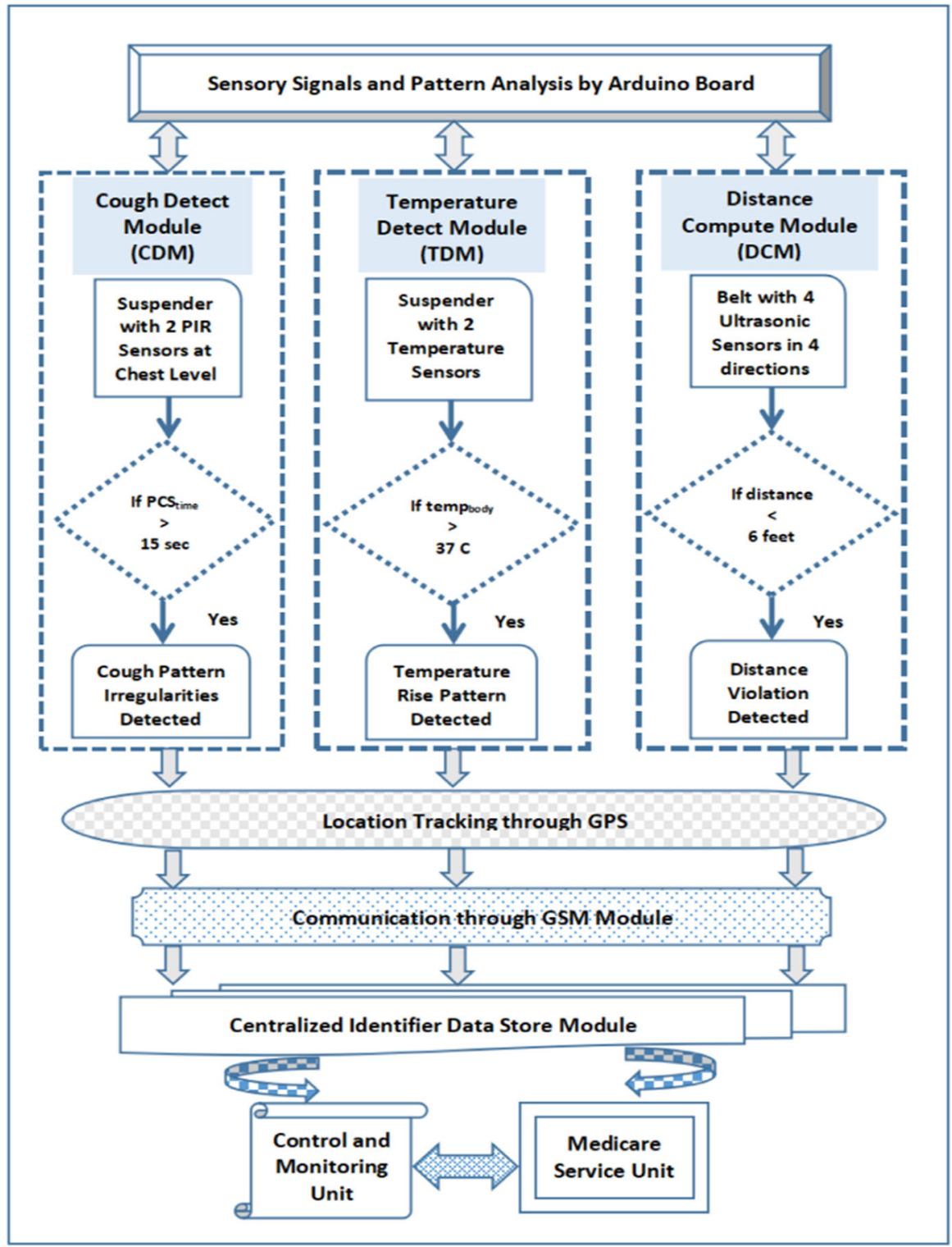

Fig. 7 Functional units of proposed Smart COVID-Shield

30 min interval. Accordingly based on these parameters, timely action can be taken by the organization.

- Data-Export Unit: Information collected from dashboard unit is aggregated in excel sheet and verified for its consistency. Ambiguities like presence of null values, redundancies, extraneous values and other errors are figured out if any and rectified. 


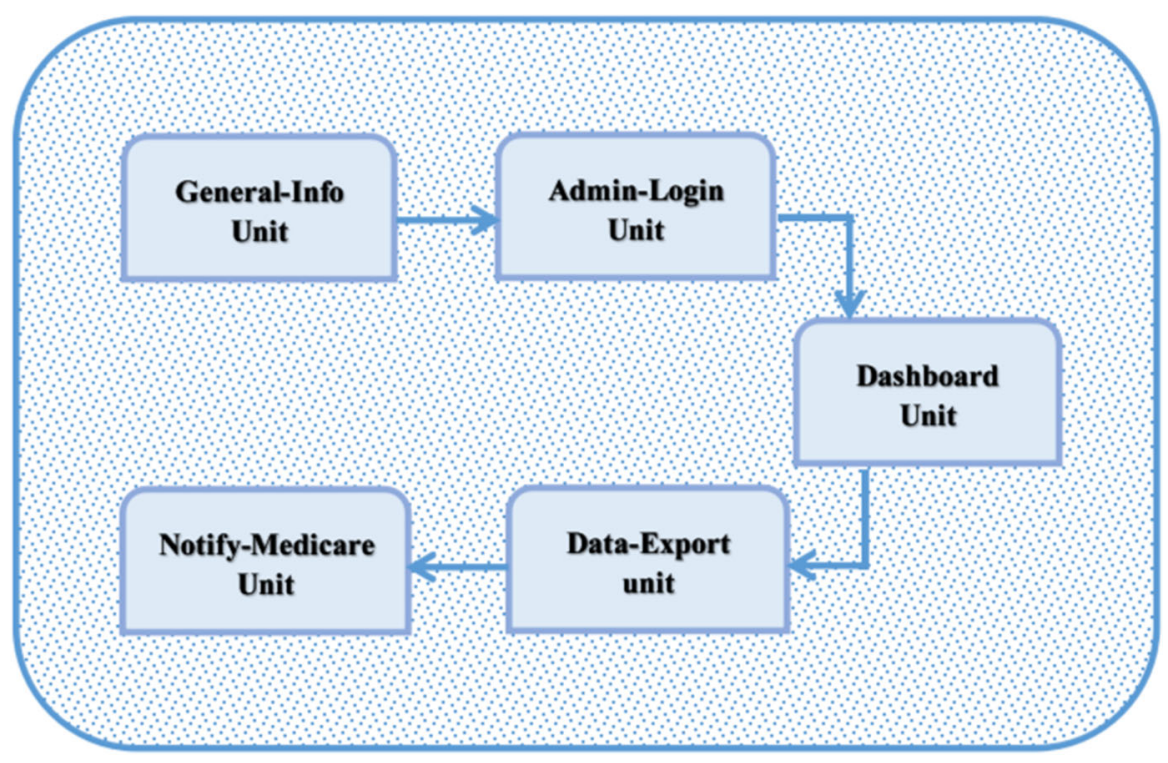

Fig. 8 Graphical interface work flow of Smart COVID-Shield

The cumulative updated excel sheet is then forwarded to the nearby medical centers for appropriate action.

- Notify-Medicare Unit: Based on the information captured by dashboard unit, the nearby local clinical centers are notified about any potential COVID-19 cases in real time basis. All data from dashboard unit is aggregated and verified for any ambiguities. Once it is validated to be consistent, it is automatically sent to medical centers without further delay. Medical staffs upon receiving the information rushes towards to the site to check-up on the detected persons and timely medical attention can be given to them.

\section{Results and analysis}

Performance of a smart model is dependent on its real time implementation to verify its effectiveness and reliability. The developed Smart COVID-Shield model was practically incorporated at an Aluminum Ingots factory named "Fe mercantile" in India to detect its capability to identify COVID-19 symptoms. Several staffs and volunteers participated in testing which was undertaken for 9 weeks. Overall analysis was separated into five distinct age groups and the testing outcome is highlighted in Table 6. Social distancing, persistent coughing and high body temperature were the parameters taken into consideration. Every week reasonable number of participants took part in the task. Week 1 records a relatively less participants with 678 people as it is the first instance the model is introduced to public. Not only week 6 has the highest participant count of 835 but also, a maximum suspected population rate of 
Table 6 Suspected population analysis affected with COVID-19

\begin{tabular}{llll}
\hline Week \# & Participants & Suspected & Suspected population $(\%)$ \\
\hline 1 & 678 & 45 & 6.63 \\
2 & 569 & 56 & 9.84 \\
3 & 720 & 69 & 9.58 \\
4 & 712 & 74 & 10.39 \\
5 & 788 & 80 & 10.15 \\
6 & 835 & 100 & 11.97 \\
7 & 823 & 74 & 8.99 \\
8 & 785 & 60 & 7.64 \\
9 & 756 & 62 & 8.2 \\
\hline
\end{tabular}

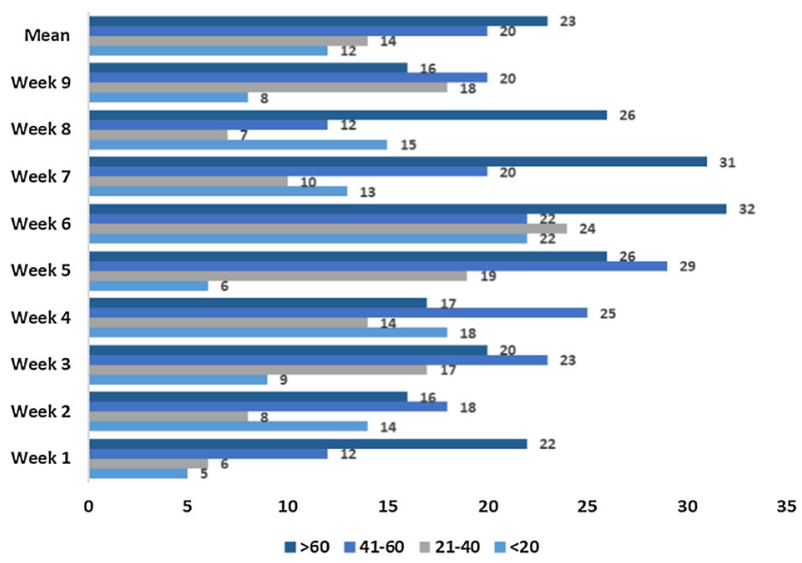

Fig. 9 Age group analysis of COVID-19 risks using proposed model

$11.97 \%$ was determined in the same week. In general, it is observed the suspected population is on higher side during week 4, 5 and 6 with $10.39 \%, 10.15 \%$ and $11.97 \%$ respectively. Week 1 shows the least participant proportion of $6.63 \%$ with suspected cases for COVID-19.

As far as varying age groups are concerned, participants with more than 40 age group exhibit higher tendency to be affected with COVID-19 symptoms. Week 5, 6 and 7 records a higher number of elderly candidates with suspected symptoms. Children and youths below 20 years of age falls in the least affected list. The average mean suspected population for beyond 60 years age group is the highest value of 23 while the average mean value for youths with less than 20 years age exhibits the least suspected candidates value of 12 persons only as seen in Fig. 9.

Body temperature is a vital symptom and plays an important role in determining a COVID-19 affected patient. At present devices available in market domain like thermal gun also, can detect high body temperature but not on continuous basis. It is suited for 


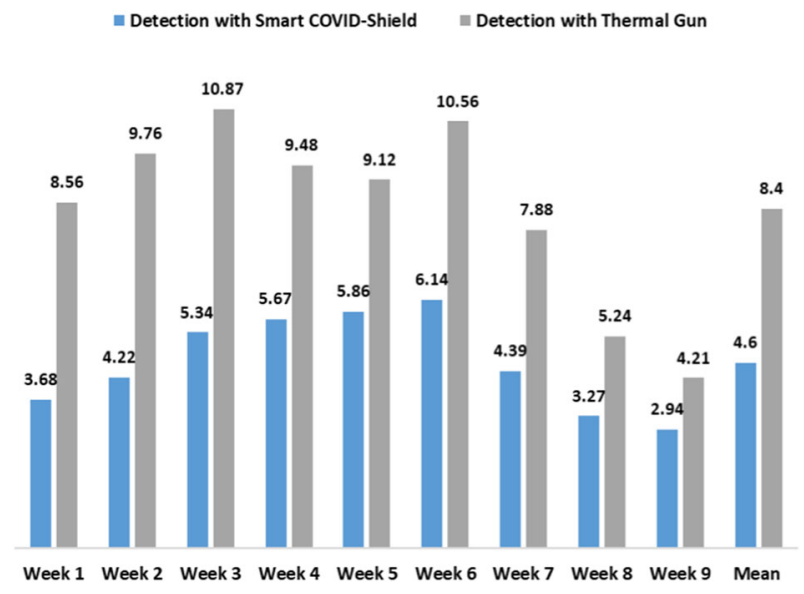

Fig. 10 COVID-19 suspects comparison using proposed model and thermal gun

a one-time temperature detection. But in several instances, it is found that candidates coming from outside may record higher temperature due to external environmental conditions like in day time sunlight. In such scenarios, continuous tracking of body temperature is crucial to exactly find the temperature fluctuation. Thus, in such cases, the developed Smart COVID-Shield is quite fruitful and reliable. An analysis was carried out using the developed Smart COVID-Shield to figure out participants with a consistent higher body temperature more than 37 degrees Celsius. As observed in Fig. 10, week 6 recorded a maximum proportion of $6.14 \%$ participants with a higher body temperature when detected with Smart COVID-Shield. A least value of $2.94 \%$ participants were identified with high body temperature on week 9 using the developed model. Interestingly when the body temperature symptom is used for analysis with both the developed model as well as thermal gun, it was seen that the number of participants almost gets doubled when detected using thermal gun device. The mean participants detected with the developed model was only $4.6 \%$ as compared to $8.4 \%$ with thermal gun.

Coughing is a general risk factor during COVID-19 observed in many affected patients. An analytical study was undertaken to identify people with persistent coughing symptom using Smart COVID-Shield. A coughing observed for more than $15 \mathrm{~s}$ and lasting for as long as $30 \mathrm{~s}$ or more is regarded as persistent coughing symptom. A maximum persistent coughing activity among $8.59 \%$ candidates is noted in week 6 while a minimum of $3.21 \%$ participants is observed in week 1 of study. The results and findings are summarized in Fig. 11.

During this pandemic period, social distance norms are implemented in almost all nations throughout world. Some people strictly follow it while others are casual about it. As per World Health Organization (WHO), maintaining a 6 feet distance reduces the speed of virus transmission and infection in public places. This social distancing norm of 6 feet distance is configured within the Smart COVID-Shield. When anyone comes too close to another thereby violating 6 feet distance then a alarm is activated with a 
Visitors (\%) with continuous coughing

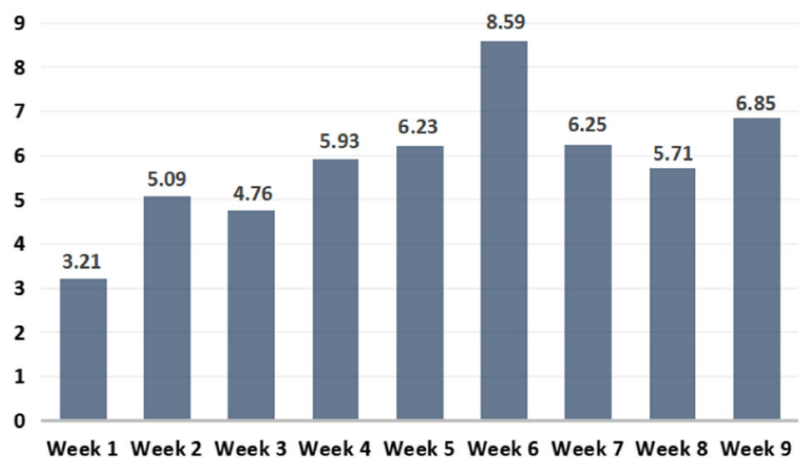

Fig. 11 Continual coughing comparative analysis over 9 weeks using proposed model

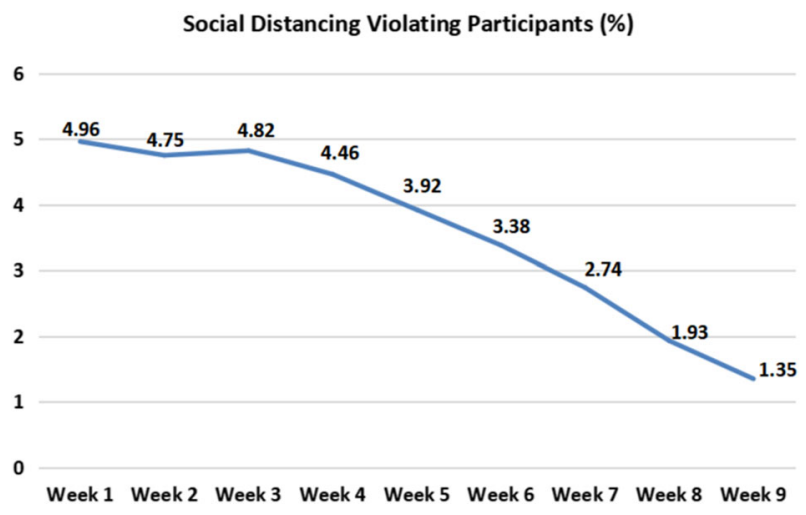

Fig. 12 Weekly analysis of social distance violating participants using proposed model

beep sound to alert the user of the violation act. As per the observations, it is clearly seen that during the initial days, more people violated the 6 feet distance norms. Lack of awareness among participant was the chief reason for the casual attitude. But as days passed by, the developed prototype model alarmed the participants and as a result people became more informed and self-aware regarding it. During the first 5 weeks of detection task, an average mean of $4.27 \%$ participants were found to violate social distancing guidelines. With passage of time, the Smart COVID-Shield managed to bring awareness among masses and as a consequence only $2.35 \%$ participants were identified to violate the 6 feet distance norm during last four weeks of analysis. A significant drop of $3.61 \%$ was observed throughout the implementation on last week. The results are shown in Fig. 12.

Execution time period is a critical factor in determining the efficiency and scalability of a smart IoT model. Especially when dealing with a critical and real-time disease diagnosis task like COVID-19 symptoms, speed of execution is utmost important. The execution latency time in the developed model sums up as the time taken to detect the 
12

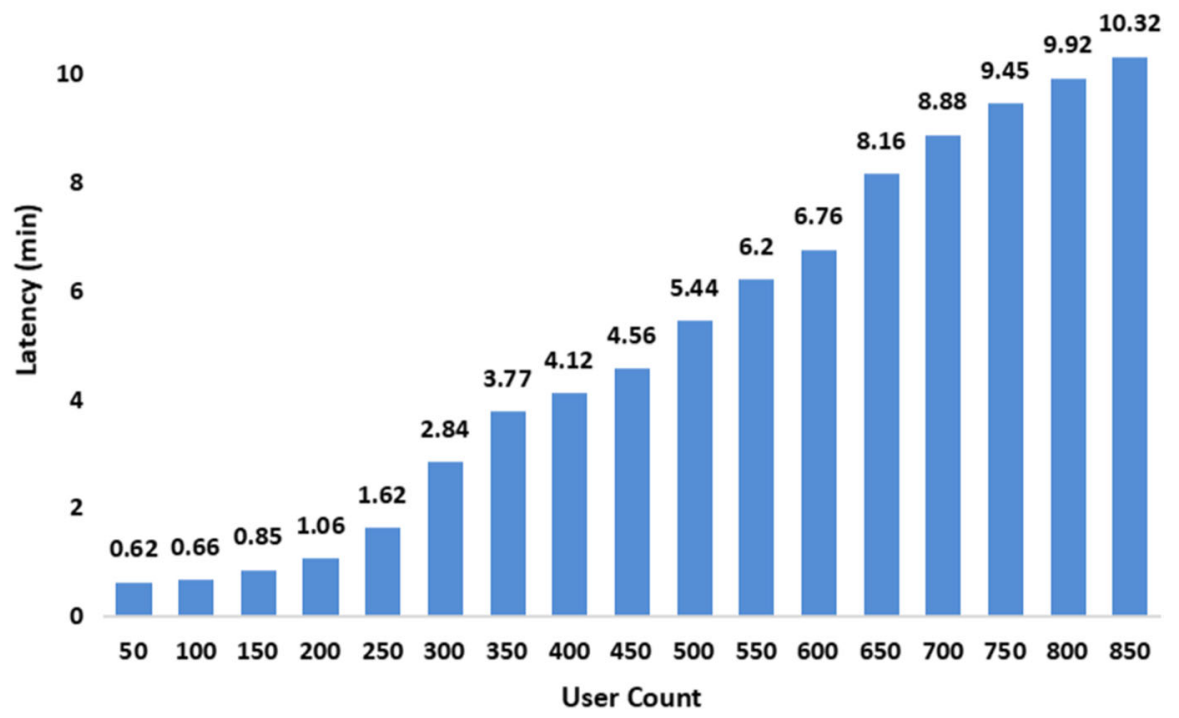

Fig. 13 Latency delay period analysis with respect to participant count

corresponding symptoms of participants, aggregate the details in an excel sheet and forward it to the nearby medicare centers. Figure 13 highlights the scalability analysis with respect to the execution time latency delay increasing the participant count at every round of execution. A slight rise in execution latency time is observed with a sharp increase in number of participants. But still with over 800 participants, the rise is still under control and is not exponential. It started with only around 50 candidates and the execution time is just $0.62 \mathrm{~min}$. When the participants count has crossed over 800 , the latency period of $10.32 \mathrm{~min}$ is still very much manageable.

Overall efficiency of any newly designed model is determined by the evaluation of domain experts. The developed Smart COVID-Shield was also validated with some healthcare domain professionals. Accuracy, connectivity, reliability and effectiveness are the evaluation metrics taken into consideration on a evaluation scale of 5. On basis of the cumulative values of these parameters, final recommendation decision is taken by experts. The outcome of evaluation is highlighted in Table 7. As it is noted in the table, as many as 6 professionals have recommended the device based on values recorded of various parameters.

The designed Smart COVID-Shield device is developed as a reliable and precise COVID-19 risks tracking model and is extremely visionary and portable. Keeping in mind that this innovation reaches out to a broader society domain, this innovation is quite affordable with an approximate setting price retained at around 34 USD (2500 INR) only. Also if this product arrives on mass production scale, the price is expected to reduce further reduce by $20 \%$. 
Table 7 Model evaluation outcome by domain professionals

\begin{tabular}{llllll}
\hline $\begin{array}{l}\text { Domain } \\
\text { expert }\end{array}$ & $\begin{array}{l}\text { Device } \\
\text { accuracy }\end{array}$ & $\begin{array}{l}\text { Module } \\
\text { connectivity }\end{array}$ & $\begin{array}{l}\text { Device } \\
\text { reliability }\end{array}$ & $\begin{array}{l}\text { Model } \\
\text { effectiveness }\end{array}$ & $\begin{array}{l}\text { Expert } \\
\text { recommend } \\
(\mathrm{Y} / \mathrm{N})\end{array}$ \\
\hline E1 & 4 & 4 & 4 & 4 & $\mathrm{Y}$ \\
E2 & 3 & 4 & 3 & 3 & $\mathrm{Y}$ \\
E3 & 3 & 3 & 3 & 3 & $\mathrm{~N}$ \\
E4 & 4 & 4 & 4 & 3 & $\mathrm{Y}$ \\
E5 & 3 & 3 & 3 & 4 & $\mathrm{~N}$ \\
E6 & 4 & 4 & 4 & 3 & $\mathrm{Y}$ \\
E7 & 4 & 3 & 4 & 4 & $\mathrm{Y}$ \\
E8 & 4 & 4 & 3 & 3 & $\mathrm{Y}$ \\
\hline
\end{tabular}

\section{Conclusion}

When the entire society is struggling with the pandemic, IoT can be successfully used to fight against it. Early detection of the corona virus symptoms is one feasible means to restrict the spreading of corona virus. An innovative real-time early detection and tracking of the pandemic symptoms using "Smart COVID-Shield" is developed and implemented in this study. It includes a suspender to detect coughing and fever along with a belt to detect social distance violators. The developed model is successfully implemented at an Aluminum factory in India over a period of nine weeks to determine its functionalities and usage. People with different age groups actively participated in the validation task. The mean suspected infect population for beyond 60 years age group was found to be optimal value of 23 while for youths, it exhibited the least mean value of 12 persons only. It was noted that week 6 recorded a maximum proportion of $6.14 \%$ participants with a higher body temperature and a least of $2.94 \%$ participants with higher body temperature was identified on week 9 using the developed model. Also, the detected suspects with "Smart COVID-Shield" were more accurate and faster when compared to detection with thermal gun. Persistent dry coughing was also effectively tracked over the weeks. Over time, social distancing guideline violators count also got reduced with the usage and awareness of this smart model and recorded a drop of $3.61 \%$. The execution latency period was also manageable with the rise in participants which makes the system scalable and reliable. Thus, it can be viewed as a promise and potential to fulfil demands from the healthcare system in efficient tracking and monitoring COVID-19 crisis. Also, it can be successfully applied in crowded places, educational institutes and several other domains to track the spread of this pandemic until and unless an efficient vaccine arrives in market. In future all other feasible symptoms can be incorporated in the model to make it more effective and real time. An emergency alert module can also be included as part of the model to create awareness among people. An enhanced security mechanism can be further embedded in the working model to prevent any data compromise and dilution in data availability. 


\section{References}

1. Singh S, Singh R, Singh KP, Singh V, Malik YP, Kamdi B, Singh R, Kashyap G (2020) Immunohistochemical and molecular detection of natural cases of bovine rotavirus and coronavirus infection causing enteritis in dairy calves. Microb Pathog 138:103814

2. Xie C, Jiang L, Huang G, Pu H, Gong B, Lin H, Ma S, Chen X, Long B, Si G, Yu H (2020) Comparison of different samples for 2019 novel coronavirus detection by nucleic acid amplification tests. Int J Infect Dis 93:264-267

3. Shen M, Zhou Y, Ye J, AL-Maskri AA, Kang Y, Zeng S, Cai S (2020) Recent advances and perspectives of nucleic acid detection for coronavirus. J Pharm Anal 10(2):97-101

4. World Health Organization, (2020) Laboratory testing for 2019 novel coronavirus (2019-nCoV) in suspected human cases, 2019: 1-7

5. Corman V, Eckerle I, Bleicker T, Zaki A, Landt O, Eschbach-Bludau M, van Boheemen S, Gopal R, Ballhause M, Bestebroer T, Muth D (2012) Detection of a novel human coronavirus by real-time reverse-transcription polymerase chain reaction. Eurosurveillance 17(39):1-6

6. Mishra S, Mallick PK, Tripathy HK, Chae GS, Mishra BSP (2021) Impact of AI and data science in response to coronavirus Pandemic. Springer, Singapore

7. Li Q, Guan X, Wu P, Wang X, Zhou L, Tong Y, Ren R, Leung KS, Lau EH, Wong JY, Xing X (2020) Early transmission dynamics in Wuhan, China of novel coronavirus-infected pneumonia. N Engl J Med 382:1199-1207

8. Zu ZY, Jiang MD, Xu PP, Chen W, Ni QQ, Lu GM, Zhang LJ (2020) Coronavirus disease 2019 (COVID-19): a perspective from China. Radiology 296(2):15-25

9. Mishra S, Sahoo S, Mishra BK (2019) Addressing security issues and standards in Internet of things. In: Mallick PK, Borah S (eds) Emerging trends and applications in cognitive computing. Hershey, IGI Global, pp 224-257

10. Mishra S, Mishra BK, Tripathy HK, Dutta A (2020) Analysis of the role and scope of big data analytics with IoT in health care domain. In: Balas VE et al (eds) handbook of data science approaches for biomedical engineering. Academic Press, United States, pp 1-23

11. S. L. Fong, D. Wui Yung Chin, R. A. Abbas, A. Jamal, and F. Y. H. Ahmed, (2019) Smart city bus application with QR code: a review. In: proceedings 2019 IEEE international conference automation control intelligent systems I2CACIS 2019, June, pp. 34-39

12. García-Valls M, Calva-Urrego C, García-Fornes A (2018) Accelerating smart eHealth services execution at the fog computing infrastructure. Future Gener Comput Syst. https://doi.org/10.1016/j.future. 2018.07.001

13. Korell F, Giannitsis E, Merle U, Kihm LP (2020) Analysis of symptoms of COVID-19 positive patients and potential effects on initial assessment. Open Access Emergency Med OAEM 12:451-457. https:// doi.org/10.2147/OAEM.S275983S

14. Islam R, Kwak D, Kabir MH, Hossain M, Kwak K-S (2015) The Internet of Things for health care: a comprehensive survey. IEEE Access 3:678-708

15. S. D. Chamberlain, I. Singh, C. A. Ariza, A. L. Daitch, P. B. Philips, and B. D. Dalziel, (2020) Real-time detection of COVID-19 epicenters within the United States using a network of smart thermometers. medRxiv

16. Tamura T, Huang M, Togawa T (2018) Current developments in wearable thermometers. Adv Biomed Eng 7:88-99

17. Mohammed M, Syamsudin H, Al-Zubaidi S, AKS RR, Yusuf E (2020) Novel COVID-19 detection and diagnosis system using IoT based smart helmet. Int J Psychosoc Rehabil 24(7):2296-2303

18. Mohammed M, Hazairin NA, Syamsudin H, Al-Zubaidi S, Sairah A, Mustapha S, Yusuf E (2020) 2019 novel coronavirus disease (COVID-19): Detection and diagnosis system using IoT based smart glasses. Int J Adv Sci Technol 29(7):954-960

19. Singh VK, Chandna H, Kumar A, Kumar S, Upadhyay N, Utkarsh K (2020) IoT-Q-Band: a low cost Internet of Things based wearable band to detect and track absconding COVID-19 quarantine subjects. EAI Endors Trans Internet Things 6(21):163997

20. Tripathy AK, Mohapatra AG, Mohanty SP, Kougianos E, Joshi AM, Das G (2020) Easyband: a wearable for safety-aware mobility during pandemic outbreak. IEEE Consum Electron Mag 9(5):57-61

21. Contact tracing IoT solution, https://bit.ly/2B60rF2, 2020, Accessed June 06, 2020

22. Aarogyasetu, https://www.mygov.in/aarogya-setu-app/, Accessed June 06, 2020 
23. Bai L, Yang D, Wang X, Tong L, Zhu X, Zhong N, Bai C, Powell CA, Chen R, Zhou J et al (2020) Chinese experts consensus on the Internet of Things-aided diagnosis and treatment of coronavirus disease 2019 (COVID-19). Clinical eHealth 3:7-15

24. COVID-19 smartphone testing kit, https://www.detectachem.com/index.php?p=COVID19, 2020, Accessed June 27, 2020

25. United against coronavirus! Stopcorona App," https://stopcorona.app/, 2020, Accessed June 06, 2020

26. Selfie app' to keep track of quarantined poles, https://www.france24.com/en/20200320-selfie-app-tokeep-track-ofquarantined-poles, 2020, Accessed June 19, 2020

27. T. Wright, Blockchain app used to track COVID-19 cases in Latin America, https://cointelegraph.com/ news/blockchain-app-used

28. TraceTogether, safer together, https://www.tracetogether.gov.sg/, 2020, Accessed June 06, 2020.11

29. Tokenpost, IoT blockchain platform launches a COVID-19 contact tracing app, https://bit.ly/3eS2VGt, 2020, Accessed June 27, 2020.11

30. S. T. Stub, () Israeli phone apps aim to track coronavirus, guard privacy, https://www.usnews.com/ news/best-countries/articles/2020-04-20/new-tech-apps-in-israel-aim-to-track-coronavirus-guardprivacy, 2020, Accessed June 27

31. M. Sharma, (2020) How drones are being used to combat COVID-19, https://www.geospatialworld. net/blogs/how-drones-are-being-used-tocombat-covid-19/,2020, Accessed June 15

32. C. Pan, (2020) Spains military uses DJI agricultural drones to spray disinfectant in fight against COVID-19, https:/www.scmp.com/tech/gear/article/3077945/spains-military-uses-djiagricultural-dronesspray-disinfectant-fight, 2020, Accessed June 15

33. J. Yang, (2020) 3 ways china is using drones to fight coronavirus, https://www.weforum.org/agenda/ 2020/03/three-ways-chinais-using-drones-to-fight-coronavirus/,2020, Accessed June 15

34. Cyient, (2020) Cyient provides drone-based surveillance technology to support telangana state police in implementing COVID-19 lockdown, https://www.cyient.com/prlisting/corporate/cyient-providesdronebased-surveillance-technology-to-support-telangana-state-police-inimplementing-covid-19lockdown, 2020, Accessed June 15

35. D. Gascuea, (2020) Drones to stop the COVID-19 epidemic, https://www.bbva.com/en/drones-to-stopthe-covid-19-epidemic/, 2020, Accessed June 15

36. Delhi civic body begins thermal screening people on balconies with drones, https://www.ndtv. com/delhi-news/coronavirus-delhi-civicbody-using-drones-to-check-temperature-of-people-onbalconies2209832, 2020, Accessed June 15, 2020

37. China buys danish robots to fight coronavirus, https://www.businesswire.com/news/home/ 20200219005708/en/ChinaBuys-Danish-Robots-Fight-Coronavirus, 2020, Accessed June 06, 2020

38. Yang G, Lv H, Zhang Z, Yang L, Deng J, You S, Du J, Yang H (2020) Keep healthcare workers safe: application of teleoperated robot in isolation ward for COVID-19 prevention and control. Chin J Mech Eng 33(1):1-4

39. Robots help combat COVID-19 in world, and maybe soon in India too, https://bit.ly/3flejtU, 2020, Accessed June 06, 2020

40. PARO therapeutic robot, http://www.parorobots.com/, 2020, Accessed July 10, 2020

41. https://www.webmd.com/lung/covid-19-symptoms\#1

42. S. S. Hassan, R. K. Rout, K. S. Sahoo, N. Jhanjhi, S. Umer, T. A. Tabbakh, and Z. A. Almusaylim, (2021) A vicenary analysis of SARS-CoV-2 genomes. Cmc-Comput Mater Continua, 3477-3493

43. Rout RK, Hassan SS, Sheikh S, Umer S, Sahoo KS, Gandomi A (2021) Feature-extraction and analysis based on spatial distribution of amino acids for SARS-CoV-2 Protein sequences. Comput Biol Med. https://doi.org/10.1016/j.compbiomed.2021.105024

Publisher's Note Springer Nature remains neutral with regard to jurisdictional claims in published maps and institutional affiliations. 